\title{
A hami melon flavor creation
}

\author{
Genfa YU ${ }^{1}$, Guangyong ZHU ${ }^{1 *}$ (D), Xiongjian ZHENG²
}

\begin{abstract}
Hami melon fruit is highly appreciated for its unique flavor. How to simulate Hami melon flavor has rarely been reported. If the Hami melon flavor is developed, it can be widely used in food, beverages, and other products. Thus, Hami melon flavor creation is of great interests. This study dealt with Hami melon flavor creation in the light of note method. The notes for construction of a Hami melon flavor were obtained by tasting and smelling Hami melon fruity. Green note, sweety note, melon fruity note, other fruity note, winey note, and special note together form Hami melon flavor. Based on these notes, a series of aroma materials were selected to construct Hami melon flavor formula. After olfactory evaluation, numerous adjustments and modifications were made until a desired Hami melon flavor formula was obtained. The flavor blended based on the formula has the typical characteristic odor of natural Hami melon fruit. It has a fresh, sweet, harmonious Hami melon aroma.
\end{abstract}

Keywords: hami melon; flavor formula; creation; aroma; sensory analysis.

Practical Application: Hami melon flavor was created and a typical Hami melon flavor formula was obtained.

\section{Introduction}

China is the largest melon-producing country and produces about $50 \%$ of the world's crop by weight (Saltveit, 2011). The production of Hami melon was 12.79 million tons in China in 2018 (Song et al., 2021). The Hami melon (Cucumis melo L.) is mainly cultivated in the Turpan-Hami basins. It is a special local product of Hami region, Xinjiang Uygur Autonomous Region, China. Due to Xinjiang's unique climatic conditions, geographical position and ecological environment, Hami melon has unique flavor and thick flesh. Because of strong sunshine and long sunshine duration, and large difference in day-night temperature, Hami melon contains high level of sugar and is rich in nutrients (Lim, 2012). The dry matter of Hami melon contains 4.6-15.8\% sugar, 2.6$6.7 \%$ cellulose (Liu et al., 2008), and has a certain quantity of malic acid, pectin, vitamins $\mathrm{A}, \mathrm{B}, \mathrm{C}$, niacin, calcium, phosphorus, and iron elements. Moshonas et al. (1993) found that the quantities of fructose, glucose, sucrose, and ascorbic acid in Hami melon were 177, 96, 96, and $0.128 \mathrm{~g} / \mathrm{kg}$ of raw melon respectively. Some bioactive compounds for health benefit, such as carotenoids, flavonoids and phenolics have also been found in melon (Wulandari et al., 2017). Usually, ripe Hami melon can be eaten fresh or made into refreshing drinks. As a kind of delicious dessert fruit, melon is enjoyed worldwide and is one of the most consumed fruit in the world (Erkmen, 2021; Perry et al., 2009). It has always been loved by the people in China. Its special flavor is much-loved by people in particular (Wang et al., 1997).

Hami melon is highly appreciated for its unique flavor (Chen et al., 2009), which is one of the most important quality indices of Hami melon (Song \& Forney, 2008). Consumers consider aroma to be one of the most important because flavor quality is the key factor for consumer acceptance. The volatile compounds create aroma and contribute to flavor (Sun et al., 2020). Because the complex mixtures of volatile chemicals result in the aroma of melons, melon volatiles have been extensively investigated (Grumet et al., 2007). Zhao et al. (2015) analyzed volatile compounds in three melon cultivars (Cucumis melo L.) by solid phase microextraction with GC-MS. They found that esters were their primary ingredients, such as butyl acetate, 2-methyl-1-butyl acetate, hexyl acetate, (Z)-3-hexenyl acetate, 2,3-butanediol diacetate, octyl acetate, benzene acetate. Wang et al. (1997) found that 3,6-nondienol, 2-pentyl-furan, and phenylmenthanol were main flavor compounds in Hami melon. Luo et al. (2019) found that some volatile sulfur chemicals are major contributors to off-flavors in heat-treated melon juice. These sulfur chemicals include 3-(methylthio)propionaldehyde, dimethyl disulfide, dimethyl sulfide, and dimethyl trisulfide. These volatile compounds are helpful to imitate Hami melon flavor. Although volatile compounds of Hami melon have been extensively studied, how to simulate Hami melon flavor has rarely been reported. If the Hami melon flavor is developed, it can be widely used in food, beverages, and other products. Therefore, Hami melon flavour creation is of great interests. Note method is an effective way to create flavors (Zhu \& Xiao, 2017; Zhu \& Yu, 2020). This study dealt with Hami melon flavor creation in the light of note method. A Hami melon flavor, with a characteristic odor of Hami melon fruit, was obtained.

\section{Materials and methods}

\subsection{Materials}

The sulfur-containing aroma compounds used in the creation of Hami melon flavor were provided by Guangzhou Levon 
Flavor and Fragrance Co. Ltd. Esters, alcohols, aldehydes, and other aroma chemicals of synthetic origin used in the creation of Hami melon flavor were obtained from Shanghai Pu-Jie Fragrance Co. Ltd. All the aroma materials were food grade, and were used as received.

\subsection{Methods}

Hami melon flavor creation was in the light of note method described in literatures (Zhu \& Xiao, 2015, 2017; Zhu \& Yu, 2020). Firstly, fresh Hami melon fruit was smelt and tasted to identify its aroma characteristics. Secondly, the knowledge of aromas and the ability to identify aromas were used to further identify and clarify which notes should be adopted to compose the Hami melon flavor to be designed. Thirdly, based on the notes of aroma materials, aroma materials with the same notes as those to compose the flavor were selected to design an initial Hami melon flavor formula. The process of the note identification of a Hami melon flavor is simply illustrated as shown in Figure 1.

These volatile compounds of melon fruit reported in literatures (Grumet et al., 2007; Neiens \& Steinhaus, 2018; Wang \& Lin, 2014; Wang et al., 1997; Zhao et al., 2015) were also considered in Hami melon flavor formula design. They are helpful for selecting aroma materials to create a Hami melon flavor. According to the initial formula, the Hami melon flavor was blended and then evaluated by smelling with smelling strips. After olfactory evaluation, numerous adjustments and modifications were made until a desired Hami melon flavor was obtained.

\section{Results and discussion}

\subsection{Notes for construction of a Hami melon flavor}

The notes for construction of a Hami melon flavor obtained by tasting and smelling Hami melon fruity were shown in Figure 2.

Green note, sweety note, melon fruity note, other fruity note, winey note, and special note together form Hami melon flavor as shown in Figure 2. A Hami melon flavor was divided into these notes. Correspondingly, these notes were used for the imitation and creation of the Hami flavor. Among these notes, fruity note is the primary note of the Hami melon flavor. The fruity note of Hami melon flavor was divided into melon fruity note and other fruity note. The melon fruity note is the aroma characteristics of a typical melon fruit. While the other

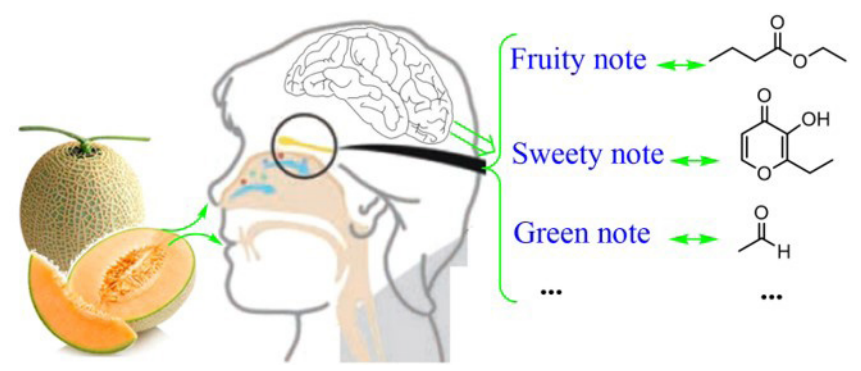

Figure 1. A simple illustration of the note identification of a Hami melon flavor. fruity note is not the aroma characteristics of the typical melon fruit but other fruits. As a modifying note, the other fruity note can provide a rich effect in melon flavor.

\subsection{Aroma materials selected to create a Hami melon flavor}

Based on the notes distinguished by tasting and smelling Hami melon fruity, a series of aroma materials were selected for construction of a Hami melon flavor. These selected aroma materials were of corresponding notes identified in Hami melon fruit as shown in Figure 2.

In the creation of Hami melon flavor, the aroma materials can be used to display green note as following: leaf alcohol, cis-3-hexenyl acetate, paracetaldehyde, acetaldehyde, and methyl octyne carbonate. These aroma chemicals make contributions to the green note of Hami melon flavor. Leaf alcohol, cis-3-hexen-1-ol, has an intensely green odor, reminiscent of freshly cut grass. It can provide natural green top note in Hami melon flavor. cis-3-hexenyl acetate, often used in combination with leaf alcohol, has a powerful, green, fruity note. It a prototype for green odor. Both paracetaldehyde and acetaldehyde have green odor. Acetaldehyde has a characteristic penetrating, green odor. Acetaldehyde is also a flavor enhancer and can help create fruitiness, juiciness and naturalness. Methyl octyne carbonate has a strong green, fatty odor. It also has a taste of unripe cucumber peel (Burdock, 2010; Surburg \& Panten, 2006). In the creation of Hami melon flavor, these aroma materials can be used for green note.

Because summer sun exposure time is long and the climate is relatively dry in Xinjiang, the Hami melon fruit produced is very sweet. High sweetness is an important characteristic of Hami melon. The aroma chemicals can be used to display sweet note in the construction of Hami melon flavor as following: maltol, ethyl maltol, furaneol, benzyl alcohol, and phenylethyl acetate. Maltol and ethyl maltol have sweet odor and often used as taste intensifier in fruit flavors. Especially, the odor of ethyl maltol is very sweet. It is four to six times stronger than maltol. Furaneol has a sweet, fruity odor and taste. Benzyl alcohol has a slightly sweet odor and a sweet taste. It is also used in flavor and fragrance as a solvent due to its relatively weak odor. Phenylethyl acetate has a honey-like undertone and a sweet, fruity taste (Burdock, 2010; Surburg \& Panten, 2006). These aroma materials can be used for sweet note in the creation of Hami melon flavor.

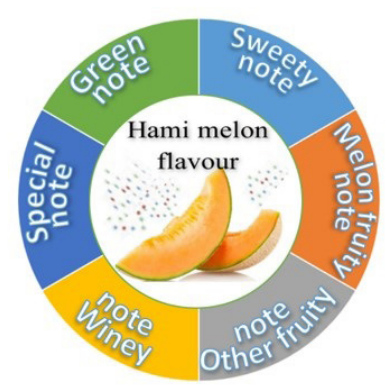

Figure 2. The notes for construction Hami melon flavor obtained by tasting and smelling Hami melon fruity. 
Melon fruity note is primarily responsible for the characteristic aroma of melon fruit. The aroma materials can be used to display melon fruity note as following: cis-6-nonenal, cis-6-nonenol, trans2-cis-6-nonadienol, and 2,6-dimenthyl-5-heptenal (synonym: melonal). cis-6-Nonenal has a melon fruity odor and taste. It also has a characteristic taste of green cucumber at $25 \mathrm{ppm}$. cis-6-Nonenol has a powerful melon odor and green odor. trans2-cis-6-Nonadienol has an intense green odor, reminiscent of cucumber and violet leaf. However, it has characteristic odor and taste of melon and watermelon at $10 \mathrm{ppm}$. It is only used in very small amounts to impart melon fruity note. Melonal has a powerful, melon, cucumber-like odor and a corresponding taste. It has a wonderful effect in imparting the character of melon fruit, an is invaluable in the creation melon (Burdock, 2010; Surburg \& Panten, 2006). These aroma materials can be used for melon fruity note in the creation of Hami melon flavor.

The other fruity note makes a contribution to the richness of the Hami melon flavor. The aroma materials can be used to display other fruity note as following: propyl acetate, butyl acetate, isobutyl acetate, isoamyl acetate, 2-methylbutyl acetate, hexyl acetate, methyl butyrate, ethyl butyrate, and ethyl levulinate. Propyl acetate has a pleasant pear-raspberry-like fruity odor. Butyl acetate has an intense fruity odor and sweet taste. Isobutyl acetate has a currant-pear-like fruity odor. Isoamyl acetate has a strong banana-like fruity odor. With a banana and apple peel-like odor, 2-methylbutyl acetate has a characteristic taste of sweet fruit at $10 \mathrm{ppm}$. Hexyl acetate has a pear-like, sweet-fruity odor. Methyl butyrate has a sweet taste and a fruity odor reminiscent of apple. With pineapple undertone, ethyl butyrate has a fruity odor and sweet taste. With a green, fruity odor, ethyl levulinate has a characteristic taste of green, melon-like fruit at $40 \mathrm{ppm}$ (Burdock, 2010; Surburg \& Panten, 2006; Wright, 2011). These aroma materials can be used for other fruity note in the creation of Hami melon flavor.

The aroma materials can be used to present winey note as following: methyl acetate, ethyl acetate, ethyl heptanoate and ethyl caprylate. With a pleasant, winey, fruity odor, methyl acetate has a rum, whiskey-like taste at $60 \mathrm{ppm}$. With a pleasant brandy-like, ethereal fruity odor, ethyl acetate has sweet, fruity taste. It is widely used in brandy and fruit flavors. Ethyl heptanoate has a winey, brandy odor and a characteristic taste reminiscent of cognac. Ethyl caprylate has a wine-apricot odor (Burdock, 2010; Surburg \& Panten, 2006; Wright, 2011). These aroma materials can be used for winey note in the creation of Hami melon flavor.

The special note for construction Hami melon flavor was odor characteristics of some volatile sulfur-containing compounds, such as 3-(methylthio) propionaldehyde (synonym: methional), dimethyl sulfide, 2-(methlythio)-ethyl acetate, dimethyl trisulfide, methyl thiobutyrate, and dimethyl disulfide. These aroma materials were used to provide special note of Hami melon flavor. They are responsible for the characteristic of authenticity. Usually, sulfur-containing compounds have potent smell and their odor threshold values are relatively low. At high concentration, the odor of these compounds is obnoxious. Therefore, methional, dimethyl sulfide, dimethyl trisulfide were seen as major contributors to off-flavors. These chemicals were believed to seriously degrade the flavor quality of melon juice (Luo et al., 2019). However, when used in high dilution, a proper amount of these compounds can present a characteristic of authenticity and provide wonderful naturalness of odor of Hami fruit. Therefore, the special note plays a crucial role in creation of Hami flavor. Methyl thiobutyrate, which was found in melon, has a putrid, sulfureous, rancid, cabbage odor. It can present tropical fruit top notes at low concentration. With potato, tomato, onion, mold ripened chesses taste characteristics at $5 \mathrm{ppb}$ to $5 \mathrm{ppm}$, methional has a strong, meat, onion-like odor. 2-(methlythio)-ethyl acetate has a fruity odor and sweet melon fruity taste at $1 \mathrm{ppm}$. Dimethyl sulfide has an unpleasant intense cabbage, wild radish odor at high concentration and has a dairy creaminess, vegetative tomato taste characteristics at $5 \mathrm{ppm}$. Dimethyl disulfide and dimethyl trisulfide have a strong odor reminiscent of onion (Burdock, 2010; Surburg \& Panten, 2006; Wright, 2011). These aroma materials can be used for special note in the creation of Hami melon flavor, and they contribute to the overall aroma perception of Hami melon flavor.

\subsection{The result of a typical Hami melon flavor formula obtained}

An initial Hami melon flavor formula was constructed by defining the ratios of the aroma materials selected. According to the initial Hami melon flavor formula designed, the Hami melon flavor was blended and the product was smelled by nose to make evaluation. Based on the result of evaluation, numerous adjustments and modifications on formula were made and Hami melon flavor samples were blended accordingly until a desired Hami melon flavor was obtained. Finally, a typical Hami melon flavor formula was obtained as shown in Table 1.

As shown in Table 1, cis-3-Hexenyl acetate, leaf alcohol, acetaldehyde and paracetaldehyde were used to impart green note in the Hami melon flavor. Among these aroma materials, cis-3-hexenyl acetate, with a powerful green and slightly fruity odor, can harmonize green note and fruity note in the Hami melon flavor. With ethanol as the solvent, acetaldehyde was used in dilution in the Hami melon flavor formula. Except for providing green note, acetaldehyde can also help create fruitiness.

Maltol, ethyl maltol, furaneol and benzyl alcohol were used to present sweet note in the Hami melon flavor. Ethyl maltol, with an intense sweet odor, was the primary ingredient for sweet note.

Cis-6-Nonenal, cis-6-nonenol, melonal, trans-2-cis-6nonadienol were used to impart melon fruity note in the Hami melon flavor. Cis-6-Nonenal, with a characteristic melon fruity odor and taste, was the main component for melon fruity note.

Propyl acetate, butyl acetate, isobutyl acetate, isoamyl acetate, 2 -methylbutyl acetate, hexyl acetate, methyl butyrate, ethyl butyrate, and ethyl levulinate were used to provide other fruity note in the Hami melon flavor. These ingredients can add enormously to the richness of the melon fruity odor. Furthermore, they can modify the harshness of acetaldehyde and paracetaldehyde (Calkin \& Jellinek, 1994).

Methyl acetate, ethyl acetate, and ethyl heptanoate were used to provide winey note in the Hami melon flavor. Winey note in combination with fruity note can convey a ripe fruity odor. 
Table 1. A typical Hami melon flavor formula.

\begin{tabular}{|c|c|c|}
\hline No. & Flavor ingredient & wt $\%$ \\
\hline 1 & Cis-3-Hexenyl acetate & 0.46 \\
\hline 2 & Leaf alcohol & 0.005 \\
\hline 3 & 40\% Acetaldehyde & 1.68 \\
\hline 4 & Paracetaldehyde & 0.012 \\
\hline 5 & Maltol & 0.012 \\
\hline 6 & Ethyl maltol & 0.46 \\
\hline 7 & Furaneol & 0.01 \\
\hline 8 & Benzyl alcohol & 0.085 \\
\hline 9 & Cis-6-Noneal & 0.33 \\
\hline 10 & Cis-6-Noneol & 0.001 \\
\hline 11 & Melonal & 0.004 \\
\hline 12 & Trans-2-cis-6-nonadienol & 0.001 \\
\hline 13 & Propyl acetate & 0.02 \\
\hline 14 & Butyl acetate & 3.09 \\
\hline 15 & Isobutyl acetate & 0.01 \\
\hline 16 & Isoamyl acetate & 3 \\
\hline 17 & 2-Methylbutyl acetate & 0.002 \\
\hline 18 & Hexyl acetate & 0.26 \\
\hline 19 & Methyl butyrate & 0.03 \\
\hline 20 & Ethyl butyrate & 1.7 \\
\hline 21 & Ethyl levulinate & 1.79 \\
\hline 22 & Methyl acetate & 0.13 \\
\hline 23 & Ethyl acetate & 3 \\
\hline 24 & Ethyl heptanoate & 0.014 \\
\hline 25 & $1 \%$ Methional & 3.93 \\
\hline 26 & $1 \%$ Methyl thiobutyrate & 3.93 \\
\hline 27 & 1\% 2-(Methylthio)-ethyl acetate & 0.04 \\
\hline 28 & $1 \%$ Dimethyl sulfide & 0.025 \\
\hline 29 & Melon base & 3 \\
\hline 30 & Ethanol & 52 \\
\hline 31 & Propylene glycol & 20.969 \\
\hline 32 & Total & 100 \\
\hline
\end{tabular}

Methional, methyl thiobutyrate, 2-(methlythio)-ethyl acetate, and dimethyl sulfide were used to impart special note in the Hami melon flavor. Because these ingredients have potent smell and their odor threshold values are relatively low, these compounds were used in dilution. Ethanol was used as the solvent for these compounds.

All aroma materials were weighed in turn according to Table 1 , and then the mixture was mixed well. Thus, the Hami melon flavor was obtained. The flavor blended based on Table 1 has the typical characteristic odor of natural Hami melon fruit. It has a fresh, sweet, harmonious Hami melon aroma.

\section{Conclusion}

This study dealt with Hami melon flavor creation in the light of note method. The notes for constructing Hami melon flavor were obtained by smelling and tasting Hami melon fruit. Hami melon flavor can be divided into green note, sweety note, melon fruity note, other fruity note, winey note, and special note. Based on these notes, a typical Hami melon flavor formula was obtained after numerous modification and adjustments. The flavor blended based on the formula has the typical characteristic odor of natural Hami melon fruit. It has a fresh, sweet, harmonious Hami melon aroma.

\section{Acknowledgements}

The authors thank the financial support from Shanghai Alliance Program (LM201844)

\section{References}

Burdock, G. A. (2010). Fenaroli's handbook of flavor ingredients. Boca Raton: CRC Press.

Calkin, R. R., \& Jellinek, J. S. (1994). Perfumery: practice and principles. New York: John Wiley \& Sons, Inc.

Chen, J., Zhang, J., Feng, Z., Song, L., Wu, J., \& Hu, X. (2009). Influence of thermal and dense-phase carbon dioxide pasteurization on physicochemical properties and flavor compounds in Hami melon juice. Journal of Agricultural and Food Chemistry, 57(13), 5805-5808. http://dx.doi.org/10.1021/jf900752b. PMid:19522496.

Erkmen, O. (2021). Bacterial inactivation mechanism of SC-CD and TEO combinations in watermelon and melon juices. Food Science and Technology. Ahead of Print. http://dx.doi.org/10.1590/fst.62520.

Grumet, R., Katzir, N. L., Little, H. A., Portnoy, V., \& Burger, Y. (2007). New insights into reproductive development in melon (Cucumis melo L.). International Journal of Plant Developmental Biology, $1(2), 253-264$.

Lim, T. K. (2012). Cucumis melo (Reticulatus Group) 'Hami melon' In T. K. Lim (Ed.), Edible medicinal and non-medicinal plants (pp. 231-234). Dordrecht: Springer.

Liu, F. 1., Wang, H. X., \& Xiao, J. (2008). Study on the processing technology of Hami melon pulp juice. Science and Technology of Food Industry, 29(8), 191-193.

Luo, D., Xu, X., Bi, S., Liu, Y., \& Wu, J. (2019). Study of the inhibitors of cooked off-flavor components in heat treated XiZhou melon juice. Journal of Agricultural and Food Chemistry, 67(37), 10401-10411. http://dx.doi.org/10.1021/acs.jafc.9b03398. PMid:31441654.

Moshonas, M. G., Shaw, P. E., Baldwin, E. A., \& Yuen, W. (1993). Volatile and nonvolatile components in Hami melon (Cucumis melo L.). Lebensmittel-Wissenschaft + Technologie, 26(6), 577-589. http:// dx.doi.org/10.1006/fstl.1993.1112.

Neiens, S. D., \& Steinhaus, M. (2018). Odor-active compounds in the special flavor hops huell melon and polaris. Journal of Agricultural and Food Chemistry, 66(6), 1452-1460. http://dx.doi.org/10.1021/ acs.jafc.7b05859. PMid:29376363.

Perry, P. L., Wang, Y., \& Lin, J. (2009). Analysis of honeydew melon (Cucumis melo var. inodorus) flavour and GC-MS/MS identification of (E,Z)-2,6-nonadienyl acetate. Flavour and Fragrance Journal, 24(6), 341-347. http://dx.doi.org/10.1002/ffj.1947.

Saltveit, M. E. (2011). Melon (Cucumis melo L.). In E. M. Yahia (Ed.), Postharvest biology and technology of tropical and subtropical fruits (pp. 31-43). Sawston: Woodhead Publishing Limited.

Song, J., \& Forney, C. F. (2008). Flavour volatile production and regulation in fruit. Canadian Journal of Plant Science, 88(3), 537-550. http:// dx.doi.org/10.4141/CJPS07170.

Song, W., Zhou, F., Shan, C., Zhang, Q., Ning, M., Liu, X., Zhao, X., Cai, W., Yang, X., Hao, G., \& Tang, F. (2021). Identification of glutathione $s$-transferase genes in Hami melon (Cucumis melo var. saccharinus) and their expression analysis under cold stress. Frontiers of Plant 
Science, 12, 672017. http://dx.doi.org/10.3389/fpls.2021.672017. PMid:34168669.

Sun, H., Chen, W., Jiang, Y., He, Q., Li, X., Guo, Q., Xiang, S., Xi, W., \& Liang, G. (2020). Characterization of volatiles in red- and whitefleshed loquat (Eriobotrya japonica) fruits by electronic nose and headspace solid-phase microextraction with gas chromatographymass spectrometry. Food Science and Technology, 40(Suppl. 1), 21-32. http://dx.doi.org/10.1590/fst.27318.

Surburg, H., \& Panten, J. (2006). Common fragrance and flavor materials: preparation, properties and uses. Weinheim: WILEY-VCH Verlag GmbH \& Co. http://dx.doi.org/10.1002/3527608214.

Wang, X., Tang, J., Tang, F., \& Ding, X. (1997). Free and glycosidically bound volatile flavor compounds in Hami melon. Nanjing Nongye Daxue Xuebao, 20(4), 93-98.

Wang, Y., \& Lin, J. (2014). Gas chromatography-olfactometry and gas chromatography-tandem mass spectrometry analysis of fresh cantaloupe (Cucumis melo L. var. cantalupensis Naudin) aroma. Flavour and Fragrance Journal, 29(2), 87-94. http://dx.doi.org/10.1002/ffj.3183.
Wright, J. (2011). Flavor Creation. Carol Stream: Allured Business Media. Wulandari, P., Daryono, B. S., \& Supriyadi. (2017). The effect of ripening stages on the antioxidant potential of melon (Cucumis melo L.) cultivar Hikapel. AIP Conference Proceedings, 1854, 020039. http:// dx.doi.org/10.1063/1.4985430.

Zhao, G., Xu, Z., Kong, W., Zhang, J., \& Xu, Y. (2015). Analysis of aromatic compounds in three melon cultivar (Cucumis melo L.) by solid phase microextraction with GC-MS. Guoshu Xuebao, 32(2), 259-266.

Zhu, G., \& Xiao, Z. (2015). Study on creation of an indocalamus leaf flavor. Food Science and Technology, 35(4), 647-651. http://dx.doi. org/10.1590/1678-457X.6770.

Zhu, G., \& Xiao, Z. (2017). Creation and imitation of a milk flavour. Food \& Function, 8(3), 1080-1084. http://dx.doi.org/10.1039/ C7FO00034K. PMid:28154863.

Zhu, G., \& Yu, G. (2020). A pineapple flavor imitation by the note method. Food Science and Technology, 40(4), 924-928. http://dx.doi. org/10.1590/fst.26019. 